\title{
Neoplastic Pericardial Disease. Analysis of 26 Patients
}

\section{Helena Nogueira Soufen, Fábio Fernandes, Barbara Maria lanni, Edmundo Arteaga, Paulo Sampaio Gutierrez, Paulo Pego-Femandes, Charles Mady}

\author{
São Paulo, SP - Brazil
}

\begin{abstract}
Purpose - To characterize patients with neoplastic pericardial disease diagnosed by clinical presentation, complementary test findings, and the histological type of tumor.
\end{abstract}

Methods - Twenty-six patients with neoplastic pericardial disease were retrospectively analyzed.

Results - Clinical manifestations and abnormalities in chest roentgenograms and electrocardiograms were frequent, but were not specific. Most patients underwent surgery. There was a high positivity of the pericardial biopsy when associated with the cytological analysis of the pericardial liquid used to determine the histological type of the tumor, particularly when the procedure was performed with the aid of pericardioscopy.

Conclusion - The correct diagnosis of neoplastic pericardial disease involves suspicious but nonspecific findings during clinical examination and in screen tests. The suspicious findings must be confirmed through more invasive diagnostic approaches, in particular pericardioscopy with biopsy and cytological study.

Keywords: malignancy, pericardial disease, pericardioscopy
Instituto do Coração do Hospital das Clínicas - FMUSP

Mailing address: Helena Nogueira Soufen - Incor - Equipe de Cardiopatias Gerais, Divisão Clínica - Av. Dr. Enéas C. Aguiar, 44 - 05403-900 - São Paulo, SP - Brazil
Pericardial disease is a malignancy complication found in $1.5 \%$ to $21.6 \%$ of the autopsies studied of several series of patients with neoplasia ${ }^{1}$. Generally, pericardial neoplastic involvement is metastatic and occurs after the diagnosis of the primary neoplasia. It is, therefore, characterized as a late complication. Pericardial involvement in neoplasia causes a higher mortality in these patients, contributing directly to death in up to $83 \%$ of the patients ${ }^{2}$. Pericardial neoplasia, in addition, is more likely to progress to cardiac tamponade, of which it is the main cause. It is, therefore, important to increase its detection, because early identification and intervention can increase patient survival. In a series of 36 patients, it was proven that delay in diagnosis contributed to premature mortality ${ }^{3}$.

Because of the importance of characterizing pericardial neoplasia, this study was performed aiming to evaluate not only the clinical presentation of patients with neoplastic involvement of the pericardium but also the histological type of the tumor, and the most frequent sites of the primary tumor, as well.

\section{Methods}

We analyzed retrospectively the records of 26 patients with neoplastic pericardial involvement. These patients were treated at the Instituto do Coração of HC-FMUSP from 1985 to 1998 . Participants included 15 men with an average age of $49.62 \pm 14.34$ years, ranging from 22 to 69 years.

These patients were chosen based on pathological findings - biopsy and autopsy - of pericardial neoplasia. Those presenting with neoplasias and pericarditis not directly related to the tumor were excluded, for example, those with pericarditis secondary to radiotherapy.

\section{Results}

The most common signs and symptoms in this group of patients were those secondary to venous congestion, 


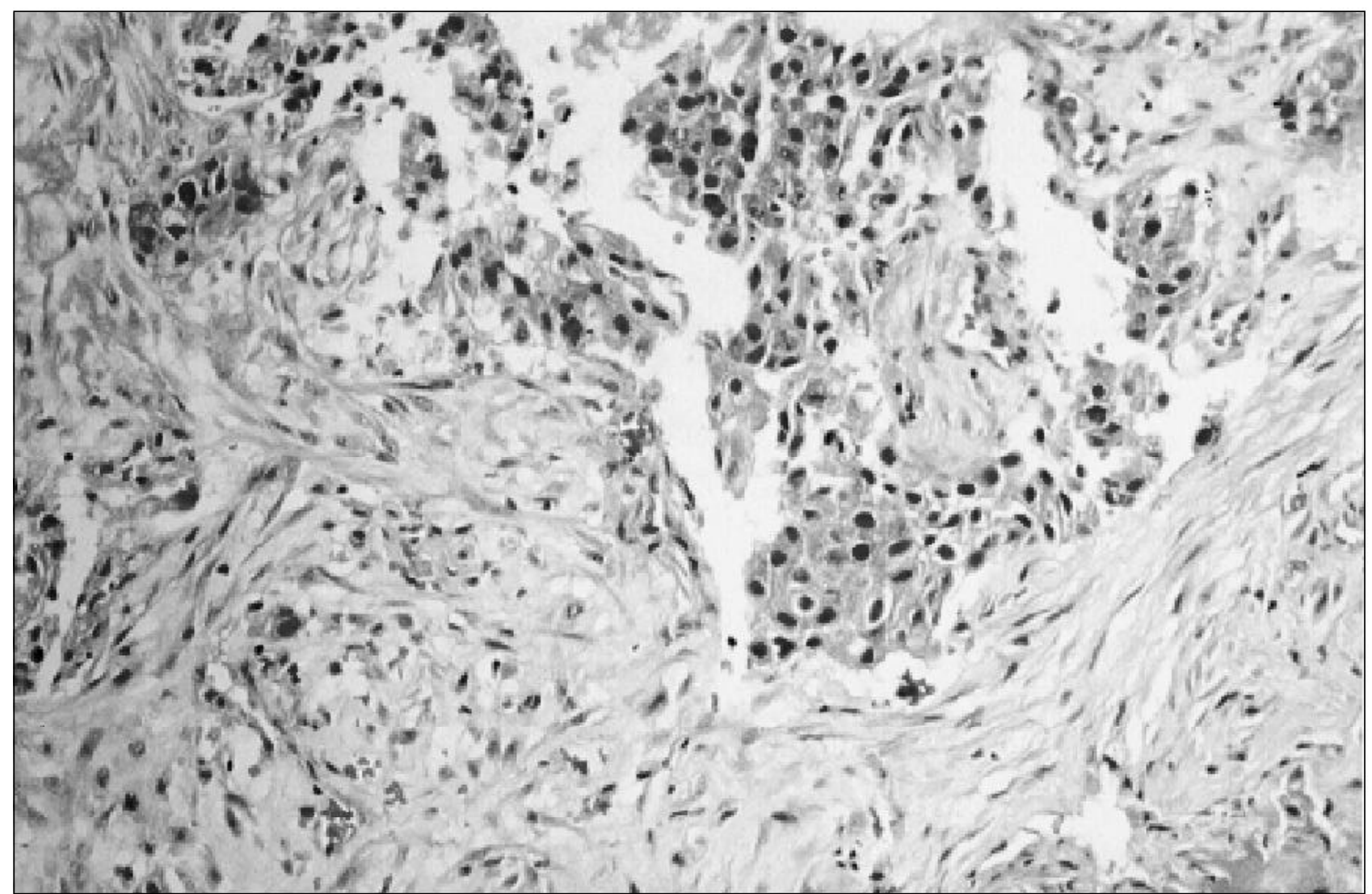

Fig. 1 - Pericardial microphotograph showing infiltration by adenocarcinoma (group of round cohesive cells presenting eosinophilic cytoplasm, at the top and in the center) with surrounding desmoplastic reaction (elongated cells amidst extracellular matrix). (HE, 10X).

\begin{tabular}{|lcc|}
\hline \multicolumn{2}{|c|}{$\begin{array}{c}\text { Table I - Most frequent signs and symptoms of patients with } \\
\text { pericardial neoplastic involvement }\end{array}$} \\
\hline \multicolumn{2}{|c|}{ Number of patients } & $\%$ \\
\hline Dyspnea & 22 & 84.6 \\
Fatigue & 18 & 69.2 \\
Lower limb edema & 18 & 69.2 \\
Chest pain & 6 & 23.1 \\
Anasarca & 4 & 15.4 \\
\hline
\end{tabular}

\begin{tabular}{|lcc|}
\hline \multicolumn{3}{|c|}{ Table II - Electrocardiographic findings } \\
\hline & Number of patients & $\%$ \\
\hline Decrease in the QRS complex & 13 & 50 \\
Amplitude in the frontal plane & 13 & 50 \\
Ventricular repolarization abnormalities & 1 & 3,8 \\
Left branch complete block & 1 & 3,8 \\
Left ventricle overload & 2 & 7,6 \\
Normal & \\
\hline
\end{tabular}

\begin{tabular}{|lcc|}
\hline \multicolumn{3}{|c|}{ Table III - Radiological findings } \\
\hline & Number of patients & $\%$ \\
\hline Isolated cardiac area enlargement & 6 & 23 \\
Pleural effusion combined with & 6 & 23 \\
cardiac area enlargement & & \\
Isolated pleural effusion & 3 & 11.5 \\
Normal & 2 & 7.6 \\
\hline
\end{tabular}

either pulmonary or systemic, as shown in table I. Six patients (26\%) presented clinical or laboratory signs of cardiac tamponade or both.

The electrocardiogram exhibited some abnormalities in the majority of the patients (22/26-84.6\%), and 13 presented low-voltage complexes in the frontal plane (table II).

The simple chest radiography revealed abnormalities in 22 patients $(84.6 \%$ ) (table III), 11 (42.3\%) presenting with an enlarged cardiac area and 7 (26.9\%) with pleural effusion. Generally, pleural effusion was associated with enlargement of the cardiac area. Only three patients (11.5\%) presented with isolated pleural effusion.

The majority of the patients studied underwent surgery (24/26-92.3\%). The most frequent procedures performed included pericardiocentesis with biopsy (15/26$57.7 \%)$, pericardioscopy with biopsy and drainage (3/26$11.5 \%)$, and tumor resection $(3 / 26-11.5 \%)$. The other approaches were sporadic and are listed in table IV.

Positivity of pericardial biopsy associated with cytology of pericardial effusion was high, around 84\% (22/26 patients). We would like to emphasize that there were no inconclusive results in the pathological exam in the three patients who underwent pericardioscopy with biopsy and drainage.

Pericardial effusion was more frequent than constrictive pericarditis (table $\mathrm{V})$. The majority of the neoplasias were metastatic (25/26-96.1\%), and among them there were 6 ca- 


\begin{tabular}{|lcc|}
\hline \multicolumn{3}{|c|}{ Table IV - Treatment } \\
\hline & Number of patients & $\%$ \\
\hline Surgical (total) & 24 & 92.3 \\
Pericardiocentesis & 15 & 57.7 \\
Pericardioscopy & 3 & 11.5 \\
Tumor resection & 3 & 11.5 \\
Partial pericardiectomy & 2 & 7.7 \\
Pleuro-pericardic window & 1 & 3.8 \\
Clinical (total) & 2 & 7.7 \\
\hline
\end{tabular}

\begin{tabular}{|lcc|}
\hline \multicolumn{3}{|c|}{ Table V - Pericardial involvement } \\
\hline & Number of patients & $\%$ \\
\hline Pericardial effusion & 22 & 84.6 \\
Constrictive pericarditis & 4 & 15.4 \\
Cardiac tamponade & 6 & 26 \\
\hline
\end{tabular}

ses of pulmonary adenocarcinoma (23\%) and 5 cases of breast adenocarcinoma (19.2\%). The other histologic types were sporadic, as shown in table VI.

Among our cases, 6 patients (26\%) had a diagnosis of carcinoma prior to the onset of cardiovascular symptoms, and 20 patients $(76.9 \%)$ had pericardial disease as the main manifestation of the neoplastic disease.

\section{Discussion}

Usually, pericardial neoplastic involvement is metastatic and manifests itself with signs of constriction in a considerable number of patients. The diagnosis may be suspected based on evidence from simple additional tests like the ECG and chest radiography, and can be confirmed by biopsy and cytology.

However, patients with neoplasia and pericardial disease do not include only those with neoplastic involvement of the pericardium. Up to $50 \%$ of patients have nonmalignant pericardial disease, such as pericarditis secondary to radiation, secondary to drugs (chemotherapy), secondary to infection, secondary to hypothyroidism, secondary to autoimmune disease, and idiopathic pericarditis.

\begin{tabular}{|lcc|}
\hline \multicolumn{3}{|c|}{ Table VI - Pathologic findings } \\
\hline & Number of patients & \% \\
\hline Metastatic neoplasias (total) & 25 & 96.1 \\
Lung adenocarcinoma & 6 & 23 \\
Breast adenocarcinoma & 5 & 19.2 \\
Lymphoma & 2 & 7.7 \\
& 1 Hodgkins & \\
Thymoma & 1 non-Hodgkins & \\
Signet-ring cell neoplasia & 2 & 7.7 \\
Kidney fibrousxanthosarcoma & 2 & 7.7 \\
Metastatic adenocarcinoma of & 1 & 3.8 \\
undetermined primary site & 7 & 26.9 \\
Primary neoplasia (mesothelioma) & 1 & 3.8 \\
\hline
\end{tabular}

Frequently, the pericardial neoplastic disease has a subclinical course, thus the in vivo diagnosis many times is not suspected ${ }^{4}$. Autopsy studies revealed that only $20 \%$ to $64 \%$ of patients had a confirmed diagnosis while still alive ${ }^{5,6}$.

In the majority of patients, chest radiography is abnormal, and pleural effusion is the most frequent feature ${ }^{6,7}$. Apparently, patients presenting pleural effusion have an enlarged cardiac area.

In our series, the most frequent symptoms were those secondary to venous congestion, showing a good correlation with other previous studies. Symptoms reported most commonly were dyspnea (81\%), chest pain (20\% to $74 \%$ ), orthopnea $(17 \%)$, and edema $(21 \%)^{1,8}$.

Most of our patients revealed electrocardiographic abnormalities (84\%), although they were of a nonspecific type. There was a high incidence of low-voltage complexes, as seen also in the literature ${ }^{5}$, reflecting a diffuse involvement.

In our series, there was a high positivity for pericardium biopsy associated with cytological analysis of the pericardial effusion (84\%). Actually, this study profile does not allow an estimation of sensitivity and specificity of biopsy and cytology performed on patients with pericardial neoplastic involvement, because these patients were chosen according to pathologic findings - biopsy and autopsy - of neoplasia in the pericardium. However, it is believed that our service's tendency to perform pericardioscopy and partial pericardiectomy could have contributed to this high positivity. Some previous studies have already demonstrated that this technical approach can increase diagnostic sensitivity. In the five patients who underwent this surgical approach, the pathological diagnosis revealed the tumor's histological type. Pericardioscopy, for example, seems to be $20 \%$ superior to conventional surgery, probably due to the visualization of the involved surfaces and a larger access to the pericardial cavity. ${ }^{6}$ The cytological analysis is also of great value, presenting sensitivity of up to $81 \%$ in some series of patients. Therefore, cytological analysis is superior to the isolated biopsy, which presents sensitivity varying only from $44 \%$ to $64 \%$. In reality, few malignant diagnoses are possible without cytology. The combination of biopsy and cytology enhances sensitivity to $90-94 \%^{4,59,10}$. We would like to emphasize that a previous study ${ }^{9}$, here performed, where pericardial biopsy was performed without the aid of pericardioscopy and without cytological analysis of the pericardial effusion, showed a biopsy positivity of $30 \%$ to $50 \%$. However, in this previous study all patients with neoplasia were considered, including those whose pericardial involvement was secondary to other morbid processes, without necessarily the direct infiltration of this serosa, as already explained.

The histological types of tumors found are similar to the ones observed in prior analyses. In order of decreasing frequency, they are lung (18\% to $37 \%$ ), breast ( $7 \%$ to $25 \%$ ), leukemia ( $10 \%$ to $20 \%$ ), lymphoma ( $8 \%$ to $21 \%$ ), and melanoma (3\% to $71 \%$ ). Among the lung carcinomas, which are the most frequent, there is a predominance of adenocarcinomas followed by epidermoid and undifferentiated carcinomas ${ }^{1,4-6}$. 
In one study, pericardial neoplastic disease resulted in cardiac tamponade in up to $46 \%$ of the patients, making it one of the main causes of this complication. In $34 \%$ to $50 \%$ of the cases, tamponade was the initial clinical presentation ${ }^{4}$.

Curiously, in our series, the majority of patients had pericardial neoplastic disease as the first manifestation of illness. However, in previous reports, this was not the common behavior of pericardial involvement ${ }^{11}$. Usually, the primary neoplasia has already been diagnosed, and the pericardial involvement is a late and metastatic manifestation. In our study, the peculiarity could be explained by the fact that the patients are usually referred to our hospital, which specializes in cardiovascular pathologies. Therefore, the patients presenting with pericardial neoplastic involvement, whose primary site is known, are possibly being treated by groups specializing in oncology. These groups, unaware of this type of complication, do not refer patients for cardiologic evaluation, either due to lack of diagnosis or to ignorance about the increase in morbidity and mortality that this type of involvement imposes on these patients.

\section{Conclusion}

Pericardial neoplastic involvement should always be suspected in patients presenting with neoplasia, because signs and symptoms are non-specific, as are general test findings. Therefore, the disease can only be diagnosed if there is a clinical suspicion. The diagnosis can be made more precise with a pericardial biopsy and cytological analysis, particularly with the aid of pericardioscopy. Diagnosis and treatment of neoplastic pericarditis is very important because, if undetected, it can cause greater mortality in those patients who have it.

\section{References}

1. Skhatsabaja LV - Secondary malignant lesions of the heart and pericardium in neoplastic disease. Oncology 1986; 43: 103-6.

2. Thurber DL, Edwards JE, Archor RWP - Secondary malignant tumors of the pericardium. Circulation 1962; 26: 228-41.

3. Markiewcz W, Borovik R, Ecker S - Cardiac tamponade in medical patients: treatment and prognosis in the echocardiographic era. Am Heart J 1986; 111 : 1138-42.

4. Cercato MC, Ferla GL, Nicoletta P, Sinto AF, Sacchi IF-Cardiac tamponade as the initial manifestation of an extracardiac malignancy. Eur J Cancer 1991; 27: 670-1.

5. Wilkes JD, Fidias P, Vaickus L - Malignancy related pericardial effusion 127 cases from the Roswell Park Cancer Institute. Cancer 1995; 76: 1377-87.

6. Kralstein J, Frishman W, Bronx NY - Malignant pericardial diseases: diagnosis and treatment. Am Heart J 1987; 113: 786-90.
7. Millaire A, Wurtz A, Groote P, Saudemont A, Chambon A, Ducloux G Malignant pericardial effusions: usefulness of pericardioscopy. Am Heart J 1992; 124: 1030-4.

8. Hancock EW - Neoplastic pericardial disease. Cardiology Clinics 1990; 8: 673-82.

9. Scully R, Mark EJ, Neely WF, Ebeling SH, Phillips LD - Case records of the Massachusetts General Hospital: a 57-year-old man. N Engl J Med 1997; 336 : 1812-9.

10. Mack MJ, Landrenau RJ, Harzlrigg SR, Acuff TE - Video thoracoscopic management of benign and malignant pericardial effusions. Chest 1993; 103 : 390S-3S.

11. Ianni BM, Pereira-Barreto AC, Mady C et al - A biópsia no diagnóstico do comprometimento pericárdico por tumores malignos. Arq Bras Cardiol 1989; 53 : $157-9$. 